\title{
A green fleet planning framework: assessment and improvement strategies
}

\author{
L. E. Teoh ${ }^{1} \&$ H. L. Khoo ${ }^{2}$ \\ ${ }^{1}$ Department of Mathematical and Actuarial Sciences, \\ Universiti Tunku Abdul Rahman, Malaysia \\ ${ }^{2}$ Department of Civil Engineering, \\ Universiti Tunku Abdul Rahman, Malaysia
}

\begin{abstract}
Under the enforcement of stringent regulations to preserve environment, airlines encounter increasing financial burden particularly in paying pollution fines. This could be avoided if airlines could improve their environmental (green) performance. This paper proposes a novel methodology to quantify airline's green performance. A 'Green Fleet Index (GFI)' framework is formulated by capturing three major environmental factors, namely aircraft emission, noise and fuel efficiency. The proposed framework also allows the evaluation of various improvement strategies to yield a greener performance. A realistic case study, with 38 international routes, is presented to examine the applicability of the developed framework. The findings show that aircraft emission, noise and fuel efficiency could affect the green performance of airlines to a great extent. Furthermore, the results point out the effectiveness of a particular mitigation strategy that performs differently on specific environmental factors (emission, noise and fuel efficiency). It is anticipated that this study may reveal some beneficial insights for airlines to operate in a sustainable and environmental manner, which would benefit air travellers as well as the community in return.
\end{abstract}

Keywords: emission, noise, fuel efficiency, sustainable transport, green performance.

\section{Introduction}

Statistically, it is reported that the net effect of nitrogen oxides emission from aircraft operations is estimated to be $24 \%$ while the carbon dioxide emission is about $2.5 \%-3 \%[1,2]$. As such, with forecasted annual air traffic growth at $5 \%$ 
[3], pollution issue will escalate to an alarming level if it is left untreated. Correspondingly, numerous local governments and airport authorities, e.g. Germany [4] have implemented stricter environmental regulations to direct airlines to be greener. Environmental fines, including emission and noise penalty, are imposed on airlines that produce excessive pollutants. Undeniably, such policies would affect airline's profit. Thus, airlines need to consider environmental issue wisely.

As reported in existing literatures, three major environmental issues pertaining to air transport system are aircraft emission, noise, and fuel consumption [5, 6]. It was found that aircraft cruising altitude, load factor, aircraft age, cabin density configuration, aircraft size, service frequency, technological innovation, fuel management strategy and aircraft taxiing operations are significant factors affecting airline's environmental performance. To alleviate deteriorating environmental problems from aircraft activities, mitigation strategies could be categorized into three major categories, namely technological innovation, operational and fleet, policy and rules and regulations [7-9]. In terms of technological innovation, improvement in engine and aerodynamics design as well as using lightweight material to reduce aircraft weight are found to be beneficial to the environment. For operational efficiency, flight and ground operations optimization are useful. Besides, lower service frequency (by operating larger aircraft) could produce lesser emission and thus larger aircraft is encouraged to retain similar capacity of demand. In terms of policies and rules and regulations, noise charge is imposed on airlines that produce excessive noise level. Generally, noise charges are imposed based on individual aircraft or cumulative noise level. Besides, emission charge is introduced to reduce aircraft emission level. The charge is generally computed based on nitrogen oxide and hydrocarbon emission level at airports. However, emission as well as noise charges vary across airports.

In brief, environmental research mainly focuses on aircraft emission, noise, and fuel consumption. Correspondingly, a wide variety of mitigation strategies is proposed to solve the relevant issues. Most of these issues and strategies are studied individually without considering the relationship among environmental factors. Thus, the real effectiveness of the strategy is not realized as the existing studies only focus on single factor. As such, there is a need to develop a suitable framework that could quantify overall green performance by capturing all influential factors. Besides, the proposed framework is able to evaluate the effectiveness of mitigation strategies on individual environmental factor.

Till to date, there are very limited studies that quantify the environmental impacts in transport sector $[10,11]$. The limitation of the existing studies is that they primarily focused on alternatives analysis and forecasting without showing any exact quantification approach to evaluate green performance. In other fields (not transport sector), fuzzy logic approach was employed [12, 13]. However, these studies are greatly depending on fuzzy membership which, in fact, does not possess a clear and specific mechanism for exact formulation. The membership functions are generally formed with the aid of some probability distribution, i.e. the results are distribution-oriented. Yet, it is important to note that for the real practice, some concerned variables may not possess specific distribution. Besides, 
Singh et al. [14] presented an overview of sustainability assessment methodologies, including environmental sustainability index. However, most of the assessment approaches generate composite index which merely based on aggregate value or weighted sum value of relevant indicators. These approaches are relatively simplistic at certain extent for which there is no clear indication on the application for more complicated problem. Furthermore, these indices did not capture uncertainty that could affect airline's green performance.

This paper aims to propose a novel methodology that is able to quantify airline's green performance. Besides, it gives insightful direction to improve green level effectively and allows airlines to investigate the effectiveness of respective mitigation strategy. The proposed approach could capture more than one environmental factor, which realistically reflects the environmental concerns in real practice. Furthermore, the proposed methodology is able to tackle the occurrence of uncertainty (e.g. adverse weather and air traffic congestion) that could affect aircraft operations. A case study is presented to evaluate the applicability of the proposed approach. It is shown that the proposed framework could yield a greener operating performance.

\section{Formulation of the Green Index (GI)}

Basically, the Green Index (GI) measures the degree of environmental performance, i.e. it is an environmental indicator which signifies the scale of green performance based on aircraft operations. The GI is derived borrowing the concept of Gini coefficient [15], which is a measure of statistical dispersion which indicates the inequality among the values of a frequency distribution. The coefficient that ranges from zero (minimum) to one (maximum) indicates equality degree from perfect equality to imperfect equality. In view of the fact that Gini coefficient measures equality degree, this signifies that a data set that is closer to each other (by having a narrower gap) would tend to have a lower Gini coefficient, and vice versa. In other words, a data set with a higher equality would have a smaller variance or standard deviation (due to a narrower gap among the data). This reveals that a smaller variance or standard variance, in fact, could reflect a greener performance. Mathematically, a data set with a higher total (or average) would result in a higher variance (or standard deviation). As such, if airlines could reduce their aircraft emission, noise and fuel consumption effectively, the resultant operating networks would produce a lesser pollutants and hence a smaller variance or standard deviation could be obtained (with a smaller average too). Equivalently, a greener performance would have a lower Gini coefficient, average, variance as well as standard deviation. As such, Gini coefficient is adopted to quantify the green level of airlines.

In this study, the GI is computed by using the geometrical approach as below:

$$
G I=1-\sum_{\forall c}\left(W_{c}+W_{c-1}\right)\left(C a t_{c}-C a t_{c-1}\right)
$$


for which $W_{c}$ indicates cumulative percentage of environmental factor $W$ in category $c$ (for vertical axis) while $\mathrm{Cat}_{c}$ denotes cumulative percentage of operating routes in category $c$ (for horizontal axis). Category, $c$, refers to the group of environmental factor (with corresponding operating routes) ranked in ascending order. Basically, GI $\rightarrow 0$ indicates that the environmental performance of airlines is greener while $G I \rightarrow 1$ implies that the green performance of airlines is getting poorer and exhibits a greater tendency to be not green. Specifically, greener fleet implies that there is a lesser amount of emission, noise and/or fuel consumption produced from aircraft operations of a particular operating year (in comparison to previous year). To quantify the overall green performance, three different indices are derived specifically for aircraft emission, noise, and fuel consumption.

\subsection{Green Emission Index $\left(G I_{E}\right)$}

The relevant contributing factors of aircraft emission are load factor, aircraft status (new or aging), emission rate and service frequency. Aircraft emission is computed during landing and take-off (LTO) cycle as well as cruising stage. In fact, emission produced from LTO and cruising stages are reported to be different. The resultant emission from LTO stage is found to be harmful as local air pollution while aircraft emission from cruising stage would result in climate change issues [7]. Aircraft emission rate, which is contributed by hydrocarbon (HC), carbon monoxide $(\mathrm{CO})$, particular matter $(\mathrm{PM})$, nitrogen oxides $\left(N O_{x}\right)$ and sulphur oxides $\left(\mathrm{SO}_{2}\right)$, i.e. major elements produced from LTO and cruising stages, is measured as total emission rate for each aircraft (in kilogram). In general, total aircraft emission of a particular operating year $t, E X_{t}$, can be expressed as follows:

$$
E X_{t}=L F_{t}\left(\sum_{\forall O D} E R_{t}^{i} F_{i, O D}^{2}\left(D_{t}, A_{t}\right) O L D+\sum_{\forall O D} E R_{t}^{i} \theta F_{i, O D}^{1}\left(D_{t}, A_{t}\right) N E W\right) \text { for } t \in T, i \in n
$$

where $L F_{t}$ refers to load factor, $E R_{t}^{n}$ is the emission rate of individual flight (depends on aircraft type $n), F_{n, O D}^{m}\left(D_{t}, A_{t}\right)$ is the service frequency of route $O D$ (depends on demand level, $D_{t}$ and total quantity of aircraft, $A_{t}$ of airline which may vary for different status of aircraft, $m$ for which $m=1$ refers to new aircraft while $m=2$ implies aging aircraft), $\theta$ is the parameter of environmental sustainability while $O L D$ and $N E W$ respectively indicates the proportion of aging (more than 1 year old) and new (up to 1 year old) aircraft servicing operating route $O D$. Particularly, total quantity of aircraft, $A_{t}$ refers to fleet size in operation for which the fleet supply is obtained via aircraft acquisition/leasing decision. The $G I_{E}$ is then computed as follows:

$$
G I_{E}=1-\sum_{\forall c}\left(E X_{t, c}+E X_{t, c-1}\right)\left(C a t_{c}-C a t_{c-1}\right) \text { for } t \in T
$$

for which $E X_{t, c}$ and $\mathrm{Cat}_{c}$ respectively denotes cumulative percentage of emission level (for vertical axis) and operating routes (for horizontal axis) in category $c$. Mathematically, $G_{E} \rightarrow 0$ signifies that the green performance of airlines in terms 
of aircraft emission appears to be better and greener for which, in overall, the servicing routes in the current operating network produce a lesser amount of emission. On the other hand, $G I_{E} \rightarrow 1$ implies that the green performance of airlines is getting poorer and exhibits a greater tendency to be not green for the current operating networks. This could happen when the operating routes emit exceptional high amount of aircraft emission which would negatively affect the overall green performance of airlines.

\subsection{Green Noise Index $\left(G I_{N}\right)$}

Generally, aircraft noise level of a particular flight can be computed based on three reference points, namely stage of lateral $\left(f_{L}\right)$, approach $\left(f_{A}\right)$ and flyover $\left(f_{F}\right)$ for which aircraft noise during these stages is greatly affected by aircraft weight (closely related to load factor) and number of engines of aircraft [16]. Accordingly, cumulative noise level, $E X N_{t}$, of a particular operating year, $t$ of airlines can be generalized as follows:

$$
E X N_{t}=L F_{t}\left(\begin{array}{l}
\sum_{\forall O D}\left(f_{L}(M)+f_{A}(M)+f_{F}(M, E)\right) F_{i, O D}^{2}\left(D_{t}, A_{t}\right) O L D \\
\sum_{\forall O D}\left(f_{L}(M)+f_{A}(M)+f_{F}(M, E)\right) \theta F_{i, O D}^{1}\left(D_{t}, A_{t}\right) N E W
\end{array}\right) \text { for } t \in T, i \in n
$$

where $f_{L}(M), f_{A}(M)$ and $f_{F}(M, E)$, respectively represents aircraft noise level at lateral, approach and flyover stages which greatly depends on aircraft weight, $M$ and number of engines, $E$. Note that annual cumulative noise level of airlines is contributed by all servicing flights and hence the service frequency of respective route, $F_{n, O D}^{m}\left(D_{t}, A_{t}\right)$ is included in eqn (4). Besides, it is important to note that aging and new aircraft might emit different noise level (mainly due to their technical specifications) and hence the status of aircraft, $m$ is considered to compute cumulative noise level. The $G I_{N}$ is then computed as follows:

$$
G I_{N}=1-\sum_{\forall c}\left(E X N_{t, c}+E X N_{t, c-1}\right)\left(C a t_{c}-C a t_{c-1}\right) \text { for } t \in T
$$

for which $E X N_{t, c}$ and $C a t_{c}$ respectively indicates cumulative percentage of noise level (for vertical axis) and operating routes (for horizontal axis) in category $c$. Basically, $G I_{N} \rightarrow 0$ implies that the green level of aircraft noise is getting better (with a tendency to be quieter or greener) by producing lesser aircraft noise throughout the operating period. Conversely, $G I_{N} \rightarrow 1$ signifies that the green level of aircraft noise is getting worst (poorer or not green). This reveals that aircraft noise is emitted substantially in the current operating networks and this would affect the overall green performance of airlines. 


\subsection{Green Fuel Efficiency Index $\left(G I_{f e}\right)$}

An aircraft that is more fuel-efficient basically utilizes less fuel for operations. Less fuel consumption for a particular fleet is relatively beneficial to airlines to travel further as well as to meet a higher level of demand. Therefore, total traveled mileage, travel demand and fuel consumption are considered to quantify the green level of fuel efficiency. For a particular operating period $t$, the fuel efficiency level, $F E L_{t}$ of airlines can be expressed as follows:

$$
F E L_{t}=L F_{t}\left(\frac{\sum_{\forall O D} g F_{i, O D}^{2}\left(D_{t}, A_{t}\right) O L D}{\sum_{\forall O D} k_{i, O D}^{2}\left(D_{t}, A_{t}\right) \sum_{\forall O D} N P_{i, O D}^{2}\left(D_{t}, A_{t}\right)}+\frac{\sum_{\forall O D} g F_{i, O D}^{1}\left(D_{t}, A_{t}\right) N E W}{\sum_{\forall O D} k_{i, O D}^{1}\left(D_{t}, A_{t}\right) \sum_{\forall O D} N P_{i, O D}^{1}\left(D_{t}, A_{t}\right)}\right) \text { for } t \in T, i \in n
$$

where $g F_{n, O D}^{m}\left(D_{t}, A_{t}\right), k_{n, O D}^{m}\left(D_{t}, A_{t}\right), N P_{n, O D}^{m}\left(D_{t}, A_{t}\right)$ respectively denotes the fuel consumption, traveled mileage and total of passenger of operating route $O D$. The $G I_{F E}$ is then computed by using the following equation:

$$
G I_{F E}=1-\sum_{\forall c}\left(F E L_{t, c}+F E L_{t, c}\right)\left(C a t_{c}-C a t_{c}\right) \text { for } t \in T
$$

for which $F E L_{t, c}$ and $C a t_{c}$ respectively refers to cumulative percentage of fuel efficiency level (for vertical axis) and operating routes (for horizontal axis) in category $c$. Similar to the concept of previous green indices, $G I_{F E} \rightarrow 0$ signifies that the environmental performance of airlines from the aspect of fuel efficiency is getting better (and greener) with less fuel consumption in overall. Conversely, $G I_{F E} \rightarrow 1$ denotes that the green level of airlines in terms of fuel efficiency tends to be poorer and not green due to extensive fuel consumption.

Compared to eqn (2) which calculates total aircraft emission, eqn (6) computes total fuel consumption of airlines. Note that in the case when more fuels are consumed, there are more aircraft emissions emitted to the environment, i.e. fuel consumption determines emission level proportionally. Contrary to aircraft emission, aircraft fuel does not have direct impacts to the environment but aircraft emission emitted through fuel burning would pose some negative consequences, e.g. local air pollution.

\section{Green Fleet Index (GFI) framework}

By considering the GI of $W$ environmental factors (aircraft emission, noise and fuel efficiency), the function of Green Fleet Index (GFI) can be quantified by making use Analytic Hierarchy Process (AHP) which is able to capture the occurrence of uncertainty [17-19]. In general, the framework to quantify GFI (for overall green performance) can be carried out as follows: 
Step 1: Obtain Green Index The relevant GI which would constitute the GFI can be obtained accordingly based on previous procedure (in section 2). Generally, major GI of airlines consists of Green Emission Index, Green Noise Index and Green Fuel Efficiency Index (as discussed earlier).

Step 2: Establish judgment matrix (for the relative comparison of GI) A pairwise comparison matrix, $A$ of GI can be expressed as follows:

$$
A=\left[\begin{array}{cccc}
1 & a_{12} & \cdots & a_{1 n} \\
1 / a_{12} & 1 & \cdots & a_{2 n} \\
\vdots & \vdots & \ddots & \vdots \\
1 / a_{1 n} & 1 / a_{2 n} & \cdots & 1
\end{array}\right]_{n \times n}
$$

for which $a_{i j}$ indicates the relative comparison of green index $G I_{i}$ over $G I_{j}$ (based on judgment scale 1-9). Generally, matrix $A$ is governed by $a_{i j} \times a_{j i}=a_{i k} \forall i, j, k$ to assure consistency.

Step 3: Calculate the largest eigenvalue As an indicator for consistency, the largest eigenvalue of a matrix can be formed as below:

$$
\lambda_{\max }=\sum_{i, j=1}^{n} a_{i j} \frac{w_{j}}{w_{i}}
$$

for which $a_{i j}$ is the element of matrix $A$ while $w_{i}$ and $w_{j}$ respectively represent the average of row $i$ and $j$ of matrix $A$. Note that a matrix is said to be more consistent if the value of the largest eigenvalue is getting closer to matrix size.

Step 4: Perform consistency test (for matrix A) To assure the consistency of matrix $A$ (with size $n$ ), consistency test can be conducted based on the consistency index, $C I$ and random consistency index, $R I$ as follows.

$$
C I=\frac{\lambda_{\max }-n}{n-1}, R I=\frac{1.98(n-2)}{n}
$$

Subsequently, the consistency ratio, $C R$ can be evaluated as follows:

$$
C R=\frac{C I}{R I}
$$

The judgment matrix is said to be consistent if $C R<0.1$. 
Step 5: Establish judgment matrix of green status (for each GI) To capture possible green status of GI (green or not), a pair-wise comparison matrix of each GI, $B_{G I_{W}}$ can be formed as follows:

$$
B_{G I W}=\left[\begin{array}{cc}
1 & S_{i j} \\
S_{j i} & 1
\end{array}\right]_{2 \times 2}
$$

for which $s_{i j}$ reflects the relative comparison of green status $s_{i}$ over $s_{j}$. Green status basically comprises two possible conditions (categories), i.e. green or not green. In fact, there is not restriction for the number of status, eqn (12) could be applied appropriately for more categories as desired by airlines.

Step 6: Perform consistency test (for matrix $B_{G I_{W}}$ ) Consistency of matrix, $B_{G^{I} W}$ can be confirmed directly by checking $a_{i j} \times a_{j i}=a_{i k} \forall i, j, k$. In fact, matrix of size $2 \times 2$ is indeed consistent in view of the fact that $a_{12} \times a_{21}=a_{11}=1$.

Step 7: Compute the GFI The resultant GFI can be evaluated as follows (where $A_{i}^{*}$ represents the average of row $i$ of normalized matrix $A$ while $B_{r}^{*}$ denotes the average of row $r$ of normalized matrix $B_{G I_{W}}$ ):

$$
G F I=\sum A_{i}^{*} B_{r}^{*}
$$

\section{Application of the GFI framework}

\subsection{Data description}

A realistic case study is set up to evaluate the applicability of the proposed methodology. It is assumed that an airline that is based in Kuala Lumpur International Airport (KLIA) is operating a total of 38 routes with five types of aircraft, namely B737-400, B737-800, B777-200, A330-300 and A380. These aircraft are chosen based on the fleet composition of Malaysia Airlines [20] in servicing international routes. To examine the green performance for a planning horizon of one year, aircraft operations are considered accordingly with the fleet size of $13,17,8,8$ and 2 respectively for the above-mentioned aircraft. The relevant data inputs, including the green performance of aircraft are compiled accordingly from accessible reports/websites [7, 16, 20-25] for which the parameter of environmental sustainability is assumed to be $\theta=96.5 \%$. A do-nothing scenario is examined by using accessible data input (without any improvement action). In addition, four improvement strategies, namely increase load factor (strategy A), operate new aircraft (strategy B), reduce service frequency (strategy C), and reduce fuel consumption (strategy D) were evaluated to examine their impacts on the green performance of airlines. Although these improvement strategies might be related to one another at certain extent, it is important to note that each strategy would be planned and implemented by airlines at different stage. 


\subsection{Results and discussions}

As shown in Table 1, it is observable that fuel efficiency is the worst performing environmental factor, followed by aircraft emission and noise. From the overall results, it could be inferred that under do-nothing scenario, airline is still relatively far from the desired green performance if there is no improvement strategy in action. Therefore, some improvement actions should be taken in accordance to current environmental performance. This could be done by focusing on fuel efficiency enhancement, particularly with the aim to reduce pollutants as well as the operational cost of airlines via fuel savings.

Besides, the results in Table 1 show that increasing load factor is an effective strategy to improve the environmental performance of airline. This strategy improves all green indices significantly, approximately to be $12.5 \%$ for all green indices, in line with the results of Miyoshi and Mason [26] and Morrell [27]. In addition, the results reveal that in average, 1\% increment of load factor would improve the green performance of airline (in terms of the GFI) up to $1.3 \%$. Generally, a higher load factor would generate a lower proportion of pollutants per unit load factor. In other words, increasing load factor is environmental beneficial. Empirically, the relation of the change of emission level and load factor could be deduced as $\Delta E X_{t}<\Delta L F_{t}$ where $\Delta E X_{t}$ and $\Delta L F_{t}$ respectively indicate the change of aircraft emission and load factor. This relation signifies that the change of emission level is relatively lower than the increment of load factor. The results, in fact, show that aircraft noise and fuel consumption exhibit similar pattern of changes. This explains the greener performance of airline in overall in terms of GFI (by increasing load factor).

Table 1: The results of illustrative case study.

\begin{tabular}{|c|c|c|c|c|}
\hline Scenario & $\begin{array}{l}\text { Green } \\
\text { Emission Index }\end{array}$ & $\begin{array}{c}\text { Green } \\
\text { Noise Index }\end{array}$ & $\begin{array}{c}\text { Green Fuel } \\
\text { Efficiency Index }\end{array}$ & $\begin{array}{l}\text { Green Fleet } \\
\text { Index (GFI) }\end{array}$ \\
\hline Do-nothing & 0.5130 & 0.3932 & 0.5562 & 0.4972 \\
\hline Strategy A & $0.4489(+12.5 \%)$ & $0.3440(+12.5 \%)$ & $0.4867(+12.5 \%)$ & $0.4351(+12.5 \%)$ \\
\hline Strategy B & $0.5077(+1.0 \%)$ & $0.3847(+2.2 \%)$ & $0.5477(+1.5 \%)$ & $0.4901(+1.4 \%)$ \\
\hline Strategy C & $0.3441(+32.9 \%)$ & $0.2860(+27.3 \%)$ & $0.3446(+38.0 \%)$ & $0.3938(+20.8 \%)$ \\
\hline Strategy D & $0.5102(+0.5 \%)$ & $0.3932(+0 \%)$ & $0.5500(+1.1 \%)$ & $0.4936(+0.7 \%)$ \\
\hline \multicolumn{5}{|c|}{$\begin{array}{l}\text { Note: } \\
\text { Strategy A: Increase load factor up to } 80 \% \text { (from } 70 \% \text { ) for entire operating networks. } \\
\text { Strategy B: Operate new aircraft for the route which exceed } 10 \mathrm{~kg} / \mathrm{km} / \text { passenger of fuel efficiency } \\
\text { (annually). } \\
\text { Strategy C: Reduce } 50 \% \text { service frequency of the route which exceeds } 10 \mathrm{~kg} / \mathrm{km} / \text { passenger of fuel } \\
\text { efficiency (annually). } \\
\text { Strategy D: Reduce } 20 \% \text { fuel consumption for entire operating networks. }\end{array}$} \\
\hline
\end{tabular}

As shown in Table 1, operating new aircraft would improve green performance of airline effectively. The results indicate that if more routes are operated with new aircraft, aircraft noise level could be improved, i.e. 2.2\% (by operating 20 routes 
with new aircraft). This is followed by the improvement of fuel efficiency and aircraft emission. In average, operating new aircraft on every 10 routes would contribute $0.7 \%$ improvement of green performance. The improvement of fuel efficiency system of new aircraft could be the major reason for a better performance of fuel efficiency and emission. Under this strategy, it was found that about $48 \%$ of total operating routes is operated with new B737-800 and 7\% of total operating networks is supported by new A380. These aircraft are claimed to be fuel-efficient $[21,27]$ and their operations in the operating networks of airline explains the promising improvement of fuel efficiency and aircraft emission. Specifically, B737-800 produces the least emission rate and A380 was claimed to consume 17\% lesser fuel (per passenger). As such, the contribution of new aircraft of B737-800 and A380 justify the comparable performance of fuel efficiency and aircraft emission. Besides, the results show that aircraft noise has the largest green improvement, i.e. about $2.2 \%$ which appears to be slightly higher than fuel efficiency and aircraft emission. This could be explained by the noise level which is emitted only from the landing and take-off (LTO) stage. Aircraft noise during cruising stage is not considered because the noise level generated from LTO stage was found to have more critical social impacts. Therefore, aircraft emission and fuel consumption which involves in both LTO and cruising stages, appear to be more (than noise).

From Table 1, the results show that reduction of service frequency is one of the constructive strategies to improve green performance. Excluding the route with single service frequency, this strategy reduces a total of 22 flights on 13 routes. As shown in Table 1, the greatest improvement (about 38\%) is on fuel efficiency index, followed by aircraft emission and noise. This is coherent with the findings of McGovern [28]. In average, green level improves approximately $9.5 \%$ for every reduction of 10 flights. The strategy to reduce service frequency implies that a particular aircraft would fly less and it was found to be effective to reduce environmental impact [29]. Comparatively, a lower quantity of flights (by reducing service frequency) would produce lesser pollutants and hence environmental impacts would be reduced proportionally. Nevertheless, it is important to note that reduction of service frequency might not be a desirable strategy for higher profit. Note that service frequency reduction may retain existing capacity of passengers (before reduction) by operating larger aircraft. This highlights that in fact the green performance of airlines (by reducing service frequency) is also closely related to fleet planning of airlines.

Airlines could reduce fuel consumption in many ways, e.g. practice optimal trajectory [30]. In this paper, fuel consumption is reduced by assuming that any approach could be applied. The results indicate that the green improvement with this strategy is relatively minimal. In terms of the fuel efficiency, ICAO [6] pointed out that an improvement of $2 \%$ over a medium planning horizon is promising and this has been set as a global target (for the year 2010-2020) to preserve the environment. With this target, it is approximated that a gradual improvement would equivalently to be $0.2 \%$ per annum. Besides, ICAO [6] revealed that a better improvement of $0.8 \%$ (per annum) is achievable with more enthusiastic actions. From Table 1, it could be seen that the findings on the green performance of airline 
in terms of fuel efficiency is practically viable as the results are very much closer to the global target, i.e. $0.2 \%-0.8 \%$ per annum. Therefore, it could be empirically confirmed that this strategy is indeed environmental beneficial for airlines and the results reflect a valid outcome.

\section{Conclusions}

In compliance to stricter environmental policies on aircraft operations, nowadays airlines have to capture green performance particularly to avoid paying tremendous fines. This could be done if airlines know their current green status and identify some effective strategies for improvement. The proposed framework in quantifying GFI in this study could assist airlines not only to quantify respective green indexes (on emission, noise and fuel efficiency) explicitly but also evaluate the effectiveness of specific mitigation strategy. In this study, three major environmental factors (emission, noise and fuel efficiency) are considered but the proposed methodology is not restricted to these factors. From the proposed strategies, it is observable that each improvement strategy has different impacts on individual environmental factor. In order to yield a greener performance, airlines may integrate numerous strategies necessarily or incorporate green issue at planning stage, e.g. include green concern in fleet planning model. Besides, it is anticipated that green improvement would contribute a higher profit level to airlines. These aspects are to be shown in future research.

\section{Acknowledgement}

The authors would like to thank the Ministry of Education (MOE) for funding the research grant FRGS under the project number FRGS/1/2012/TK08/UTAR/03/3.

\section{References}

[1] Lee, D.S., Fahey, D.W., Forster, P.M., Newton, P.J., Wit, R.C.N., Lim, L.L., Owen, B., \& Sausen, R., Aviation and global climate change in the $21^{\text {st }}$ century. Atmosphere Environment. Doi: 10.1016/j.atmosenv.2009.04.024., 2009.

[2] Anger, A., Including aviation in the European emissions trading scheme: Impacts on the industry, $\mathrm{CO}_{2}$ emissions and macroeconomic activity in the EU. Journal of Air Transport Management, 16, pp. 100-105, 2010.

[3] International Air Transportation Association (IATA). Financial forecast 2009, Montreal, 2009.

[4] Lu, C., The implications of environmental costs on air passenger demand for different airline business model. Journal of Air Transport Management, 15, pp. 158-165, 2009.

[5] Intergovernmental Panel on Climate Change (IPCC). Aviation and the Global Atmosphere. Www.grida.no./publications/others/ipcc_sr/?src=/ Climate/ipcc/aviation/index.html 
[6] International Civil Aviation Organization (ICAO). Environmental report 2010. http://www.icao.int/

[7] Givoni, M, \& Rietveld, P., The environmental implications of airlines' choice of aircraft size. Journal of Air Transport Management, 16(3), pp. 159-167, 2010.

[8] Sgouridis, S., Bonnefoy, P.A., \& Hansman, R.J., Air transportation in a carbon constrained world: Long-term dynamics of policies and strategies for mitigating the carbon footprint of commercial aviation. Transportation Research Part A, 45, pp. 1077-1091, 2011.

[9] European Civil Aviation Conference (ECAC). Recommendation ECAC/27-

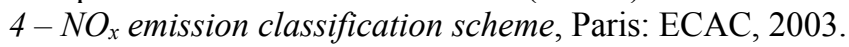

[10] Boclin, A.D.S.C., \& Mello, R.D. A decision support method for environmental impact assessment using a fuzzy logic approach. Ecological Economics, 58, pp. 170-181, 2006.

[11] Rossi, R., Gastaldi, M., \& Gecchele, G. Sustainability evaluation of transportation policies: A fuzzy-based method in a "what to" analysis. 17th Online Conference on Soft Computing in Industrial Applications, Czech Republic, December 10-21, 2012.

[12] Silvert, W., Fuzzy indices of environmental conditions. Ecological Modelling, 130, pp. 111-119, 2000.

[13] Valente, T.M., Ferreira, M.J., \& Gomes, C.L., Application of fuzzy logic to qualify the environmental impact in abandons mining sites. Water Air Soil Pollution, 217, pp. 303-315, 2011.

[14] Singh, R.K., Murty, H.R., Gupta, S.K., \& Dikshit, A.K., An overview of sustainability assessment methodologies. Ecological Indicators, 15, pp. 281-299, 2012.

[15] Gini, C. Variabilità e mutabilità (Variability and Mutability), Bologna: Tipografia di Paolo Cuppini, 1912.

[16] International Civil Aviation Organization (ICAO). Environmental protection - Volume I aircraft noise ( $6^{\text {th }}$ edition), Canada, 2011.

[17] Zadeh, L., Fuzzy sets. Information and Control, 8, pp. 338-353, 1965.

[18] Saaty, T.L., The Analytic Hierarchy Process: Planning, Priority Setting and Resource Allocation. New York: McGraw-Hill, 1980.

[19] Saaty, T.L., A scaling method for priorities in hierarchy structures. Journal of Mathematical Psychology, 15, pp. 234-281, 1977.

[20] Malaysia Airlines. http://www.malaysiaairlines.com.my

[21] Airbus. http://www.airbus.org

[22] Scheelhaase, J.D. Local emission charges - A new economic instrument at German airports. Journal of Air Transport Management, 16, pp. 94-99, 2010.

[23] Khoo, H.L., \& Teoh, L.E. An optimal aircraft fleet management decision model under uncertainty. Journal of Advanced Transportation. DOI: 10.1002/atr.1228, 2013.

[24] AirAsia Berhad. http://www.airasia.com.my

[25] Boeing. 2013. http://www.boeing.org 
[26] Miyoshi, C., \& Mason, K.J., The carbon emissions of selected airlines and aircraft types in three geographic markets. Journal of Air Transport Management, 15, pp. 138-147, 2009.

[27] Morrell, P., The potential for European aviation $\mathrm{CO}_{2}$ emissions reduction through the use of larger jet aircraft. Journal of Air Transport Management, 15, pp. 151-157, 2009.

[28] McGovern, E., Controlling the environmental impact of transport: Traffic management schemes and their influence on consumer behaviour. Sustainable Development, 6, pp. 23-30, 1998.

[29] Lijesen, M., Straaten, W.v.d, Dekkers, J., Elk, R.V., \& Blokdijk, J., How much noise reduction at airports? Transportation Research Part D, 15, pp. 51-59, 2010.

[30] Nikoleris, T., Gupta, G., \& Kistler, M., Detailed estimation of fuel consumption and emissions during aircraft taxi operations at Dallas/Fort Worth International Airport. Transportation Research Part D, 16, pp. 302-308, 2011. 